\title{
Multivitamin Analysis of Fruits, Fruit-Vegetable Juices, and Diet Supplements
}

\author{
Joanna Plonka • Agata Toczek • Violetta Tomczyk
}

Received: 30 July 2011 / Accepted: 12 December 2011 / Published online: 13 January 2012

(C) The Author(s) 2012. This article is published with open access at Springerlink.com

\begin{abstract}
Vitamins are organic compounds that are required for various biological functions. In general, vitamins are not synthesized in the human body, but lack or deficiency of them may lead to certain diseases. Determinations of 11 vitamins in various products were performed, which included ascorbic acid (C), seven vitamins of the $\mathrm{B}$ group (thiamine $\mathrm{B}_{1}$, riboflavin $\mathrm{B}_{2}$, nicotinamide $\mathrm{B}_{3}$, pantothenic acid $\mathrm{B}_{5}$, pyridoxine $\mathrm{B}_{6}$, folic acid $\mathrm{B}_{9}$, and cyanocobalamin $\mathrm{B}_{12}$ ), as well as three fat-soluble vitamins (retinol $\mathrm{A}$, cholecalciferol $\mathrm{D}_{3}$, and $\alpha$-tocopherol $\mathrm{E}$ ). A column with RP18 stationary phase and a diode array detector with properly selected analytical wavelengths for each compound were used. A gradient of trifluoroacetic acid in water with methanol was used as the mobile phase. Limits of quantification in the range of $0.70-2.90 \mu \mathrm{g} / \mathrm{mL}$ for watersoluble vitamins and $1.85-15.84 \mu \mathrm{g} / \mathrm{mL}$ for fat-soluble vitamins were obtained. Those values are sufficient for determinations of the aforementioned compounds in foodstuff. The developed procedure of sample preparation together with chromatographic system can be used for food quality monitoring in the food industry.
\end{abstract}

Keywords Water-soluble vitamins · Fat-soluble vitamins . Fruits · Juices · High-performance liquid chromatography · SPE

\section{Introduction}

Vitamins are a vital group of food components that must be provided to the human body in sufficient amounts. They

J. Płonka $(\bowtie) \cdot$ A. Toczek $\cdot$ V. Tomczyk

Department of Analytical Chemistry,

Silesian University of Technology,

7 M. Strzody Str.,

44-100 Gliwice, Poland

e-mail: joanna.plonka@polsl.pl support metabolism processes and improve the efficiency of proteins and enzymes. Vitamins are provided in final form or as provitamins that are subsequently transformed into vitamins. The main sources of vitamins are fruits, vegetables, meats, and fish. However, when acquisition from food is insufficient, the vitamins can be acquired from pharmaceutical preparations. Currently, the increased interest in a balanced and wholesome diet has caused increased demand for multivitamin diet supplements.

Vitamins are divided into two groups based on their solubility. The fat-soluble vitamins $\mathrm{A}, \mathrm{D}_{3}, \mathrm{E}$, and $\mathrm{K}$ are non-polar, hydrophobic compounds that are isoprene derivatives. They are absorbed by the human organism also with fat and can be stored in liver, kidneys, and fat tissue. Fatsoluble vitamins perform essential roles in the regulation of height, calcium and phosphorus absorption, and the development and functionality of bones. They also act as anticoagulants. The water-soluble vitamin group contains vitamin $\mathrm{C}$ (ascorbic acid), $\mathrm{B}_{1}$ (thiamine), $\mathrm{B}_{2}$ (riboflavin), $\mathrm{B}_{3}$ (nicotinamide), $\mathrm{B}_{5}$ (pantothenic acid), $\mathrm{B}_{6}$ (pyridoxine), $\mathrm{B}_{9}$ (folic acid), and $\mathrm{B}_{12}$ (cyanocobalamin). They have different chemical structures because they represent acid, pyrimidine, and imidazole derivatives as well as acid amides. They are responsible for the proper functioning of the nervous and respiratory systems, synthesis of nucleic and fatty acids, and creation of red blood cells (National Academy of Sciences 1989; Sikorski 2007). Lack or deficiency of vitamins in consumed food can lead to deficiency states and diseases.

A widely used analytical method for the determination of vitamins in food and diet supplements is chromatography, especially high-performance liquid chromatography (HPLC). HPLC is described by high resolution and selectivity, short time of analysis, and ability to couple with other techniques. Literature publications describe numerous methods for vitamin determination; however, particular 
procedures allow only determining one, two, or several vitamins at the same time, but only from either the fat- or water-soluble group. Table 1 shows parameters of chromatographic procedures for the determination of fat- and water-soluble vitamins. Literature data allow the selection of a chromatographic procedure that enables the determination of vitamins in food samples with adequate concentration levels with the use of both gradient and isocratic systems. The disadvantage of the described procedures is the small number of analytes that can be determined at the same time. Most of the methods allow the determination of several vitamins from one group at once. Most often, it is vitamin A together with $\mathrm{E}$ or three to four vitamins of the B group. Only in single particular cases does a chromatographic procedure allow for the simultaneous separation of several compounds of both fat- and water-soluble groups without requirement of stationary or mobile phase changes.

Another issue is proper food sample preparation, which would allow the simultaneous extraction of all analytes from a matrix. Solid-liquid and solid phase extraction are the most often used extraction techniques. Most of the procedures described in the literature concern single vitamins of water-soluble vitamins (Hussein et al. 2000; Kurilich et al. 1999; Wang et al. 1996; Franke et al. 2004; Frenich et al. 2005; Gil et al. 2006; Mahattanatawee et al. 2006; Wall 2006; Singh et al. 2007; Lopez-Berenguer et al. 2009) and fat-soluble vitamins (Kurilich et al. 1999; Mahattanatawee et al. 2006; Singh et al. 2007; Ching and Mohamed 2001). Only two papers (Ndaw et al. 2000; Lebiedzińska and Szefer 2006) describe procedures that allow the simultaneous extraction of three to four vitamins from one group. Other studies (Moreno and Salvadó 2000; Munzuroglu et al. 2003) show that it is possible to extract vitamins from both groups; however, this requires a few additional changes in the procedures, like adding a secondary solvent.

Our study presents results of the development of an analytical procedure for the simultaneous extraction as well as simultaneous chromatographic determination of fat- and water-soluble vitamins. After an examination of standard samples, application to real samples was performed. The developed method allows reduction in time for the analysis of fruits, vegetables, and juices, as well as multivitamin diet supplements.

\section{Materials and Methods}

\section{Reagents}

Standard solutions $(10 \mathrm{mg} / \mathrm{mL})$ of retinol (A), cholecalciferol $\left(D_{3}\right), \alpha$-tocopherol $(E)$, thiamine $\left(B_{1}\right)$, riboflavin $\left(B_{2}\right)$, nicotinamide $\left(\mathrm{B}_{3}\right)$, pantothenic acid $\left(\mathrm{B}_{5}\right)$, pyridoxine $\left(\mathrm{B}_{6}\right)$, folic acid $\left(\mathrm{B}_{9}\right)$, cyanocobalamin $\left(\mathrm{B}_{12}\right)$, and ascorbic acid $(\mathrm{C})$ (all from Sigma, St. Louis, MO, USA) were prepared in methanol. Methanol, trifluoroacetic acid (TFA), and water of HPLC grade used for the mobile phase were purchased from Merck (Darmstadt, Germany). Acetic acid 99\%, zinc acetate, and potassium ferricyanide (all from $\mathrm{POCH}$, Gliwice, Poland) of analytical grade were used. Standard solutions were prepared directly before use.

\section{High-Performance Liquid Chromatography Conditions}

HPLC analysis was performed using a Merck-Hitachi chromatograph equipped with an L6200A pump, L-7360 thermostat, and L4500A diode array detector. Chromatographic separation was carried out on a TSKGel ODS-100V, $150 \times 4.6 \mathrm{~mm}(5 \mu \mathrm{m})$ column (Tosoh Bioscience). Examination was performed at a temperature of $30{ }^{\circ} \mathrm{C}$. A gradient elution with $0.01 \%$ TFA in water (A) and methanol (B) was applied. The gradient elution parameters are shown in Table 2.

Detection of vitamins was performed using a diode array detector (DAD) with analytical wavelengths typical for each analyte: retinol, $320 \mathrm{~nm}$; cholecalciferol, $275 \mathrm{~nm}$; thiamine, $253 \mathrm{~nm}$; riboflavin, $\alpha$-tocopherol, and cyanocobalamin, $290 \mathrm{~nm}$; nicotinamide, $258 \mathrm{~nm}$; pantothenic acid, $218 \mathrm{~nm}$; pyridoxine, $289 \mathrm{~nm}$; folic acid, $360 \mathrm{~nm}$; and ascorbic acid, $262 \mathrm{~nm}$.

\section{Calibration Curves}

The calibration curves for the examined compounds were prepared in a matrix of methanolic food extract. Matrix of food samples was purified by solid phase extraction with the same parameters as the extraction of analytes (see "Sample Preparation"). The effluent formed during sample passing through solid phase was collected. Then, appropriate amounts of standard solutions were added.

Curves were determined in a concentration range appropriate for the expected content of analytes in real food samples. The number of experimental points taken for regression was $n=6$. Every analyte was injected three times. The volume of each injection was $20 \mu \mathrm{L}$. Values of standard deviation of slope and intercept for calibration curves were calculated.

\section{Carrez I and Carrez II Solution Preparation}

Carrez I solution was prepared by dissolving $10.6 \mathrm{~g}$ of potassium ferricyanide $\left(\mathrm{K}_{4} \mathrm{Fe}(\mathrm{CN})_{6} \cdot 3 \mathrm{H}_{2} \mathrm{O}\right)$ in $100 \mathrm{~mL}$ of distilled water. Carrez II solution was prepared by dissolving $21.95 \mathrm{~g}$ of zinc acetate $\left(\mathrm{Zn}\left(\mathrm{CH}_{3} \mathrm{COO}\right)_{2} \cdot 2 \mathrm{H}_{2} \mathrm{O}\right)$ and $30 \mathrm{~mL}$ of $99 \%$ acetic acid in $100 \mathrm{~mL}$ of distilled water. 
Table 1 Procedures for the selected chromatographic determination methods of vitamins in foodstuff

\begin{tabular}{|c|c|c|c|c|c|c|c|c|}
\hline No. & Vitamins & $\begin{array}{l}\text { Mobile } \\
\text { phase }\end{array}$ & $\begin{array}{l}\text { Stationary } \\
\text { phase }\end{array}$ & Detector & $\begin{array}{l}\text { LOD } \\
(\mathrm{ng} / \mathrm{mL})\end{array}$ & $\begin{array}{l}\text { Measurement } \\
\text { range }(\mu \mathrm{g} / \mathrm{mL})\end{array}$ & $\begin{array}{l}\text { Sample } \\
\text { type }\end{array}$ & Reference \\
\hline \multirow[t]{11}{*}{1} & A & A: $0.010 \%$ TFA (pH 3.9$)$ & \multirow{11}{*}{$\begin{array}{l}\text { C18. } 150 \times 4.6 \mathrm{~mm} \\
\quad(3 \mu \mathrm{m})\end{array}$} & \multirow{11}{*}{$\begin{array}{l}\text { DAD } \\
\text { MS }\end{array}$} & $12.30-12.49$ & - & \multirow[t]{11}{*}{ Vitamin preparations } & \multirow[t]{11}{*}{ Klejdus et al. (2004) } \\
\hline & $\mathrm{D}$ & B: $\mathrm{MeOH}$ & & & $41.77-129.4$ & & & \\
\hline & $\mathrm{E}$ & \multirow[t]{9}{*}{ Gradient elution $35 \mathrm{~min}$} & & & $34.78-127.30$ & & & \\
\hline & $\mathrm{K}$ & & & & $83.10-197.40$ & & & \\
\hline & $\mathrm{B}_{1}$ & & & & 12.18 & & & \\
\hline & $\mathrm{B}_{2}$ & & & & 188.00 & & & \\
\hline & $\mathrm{B}_{3}$ & & & & $16.41-35.40$ & & & \\
\hline & $\mathrm{B}_{6}$ & & & & $21.17-23.08$ & & & \\
\hline & $\mathrm{B}_{9}$ & & & & 30.48 & & & \\
\hline & $\mathrm{B}_{12}$ & & & & 97.47 & & & \\
\hline & $\mathrm{C}$ & & & & 25.55 & & & \\
\hline \multirow[t]{13}{*}{2} & $\mathrm{~B}_{1}$ & A: $0.4 \mathrm{M} \mathrm{LiClO}_{4}(\mathrm{pH} 2.4$ ) & \multirow{13}{*}{$\begin{array}{l}\mathrm{C} 18,75 \times 2 \mathrm{~mm} \\
\quad(5 \mu \mathrm{m})\end{array}$} & \multirow[t]{13}{*}{ UV } & \multirow[t]{13}{*}{-} & \multirow[t]{13}{*}{-} & \multirow[t]{13}{*}{ Vitamin preparations } & \multirow{13}{*}{$\begin{array}{l}\text { Kozhanova } \\
\text { et al. (2002) }\end{array}$} \\
\hline & $\mathrm{B}_{2}$ & B: $\mathrm{CH}_{3} \mathrm{CN}$ & & & & & & \\
\hline & $\begin{array}{l}\mathrm{B}_{3} \\
\mathrm{~B}_{5}\end{array}$ & \multirow[t]{6}{*}{ Gradient elution $25 \mathrm{~min}$} & & & & & & \\
\hline & $\mathrm{B}_{6}$ & & & & & & & \\
\hline & & & & & & & & \\
\hline & $\mathrm{B}_{7}$ & & & & & & & \\
\hline & $\mathrm{B}_{9}$ & & & & & & & \\
\hline & $\mathrm{B}_{12}$ & & & & & & & \\
\hline & A & \multirow[t]{5}{*}{$\mathrm{CH}_{3} \mathrm{CN} / \mathrm{H}_{2} \mathrm{O}(95: 5, v / v)$} & & & & & & \\
\hline & $\mathrm{D}_{2}$ & & & & & & & \\
\hline & $\mathrm{D}_{3}$ & & & & & & & \\
\hline & $\mathrm{E}$ & & & & & & & \\
\hline & $\mathrm{K}_{3}$ & & & & & & & \\
\hline \multirow[t]{8}{*}{3} & $\mathrm{~B}_{1}$ & A: $0.05 \mathrm{M} \mathrm{CH}_{3} \mathrm{COONH}_{4}$ & \multirow{8}{*}{$\begin{array}{l}\text { C18. } 150 \times 3.9 \mathrm{~mm} \\
\quad(4 \mu \mathrm{m})\end{array}$} & UV-Vis & 3.18 & $4.93-39.44$ & Vitamin preparations & Moreno and \\
\hline & $\mathrm{B}_{2}$ & $\mathrm{~B}: \mathrm{MeOH}$ & & & 1.84 & $2.18-17.43$ & & Salvadó (2000) \\
\hline & $\mathrm{B}_{3}$ & Gradient elution $17 \mathrm{~min}$ & & & 9.92 & $17.53-140.24$ & & \\
\hline & $\mathrm{B}_{6}$ & & & & 1.37 & $1.78-14.20$ & & \\
\hline & $\mathrm{B}_{12}$ & & & & 0.04 (LOQ) & $0.04-0.12$ & & \\
\hline & A & $\mathrm{CH}_{3} \mathrm{CN} / \mathrm{MeOH}$ & & & 5.00 & $4.94-39.49$ & & \\
\hline & $\mathrm{E}$ & $(95: 5 . v / v)$ & & & 3.09 & $10.15-81.20$ & & \\
\hline & D3 & & & & 0.05 & $0.16-1.30$ & & \\
\hline 4 & $\mathrm{~B}_{1}$ & A: 5 mM HFBA & $\mathrm{C} 18.250 \times 4.6 \mathrm{~mm}$ & MS & 3 & $0.01-50$ & Vitamin preparations & Chen et al. (2006) \\
\hline & $\mathrm{B}_{2}$ & B: $\mathrm{MeOH}$ & $(5 \mu \mathrm{m})$ & & 6 & $0.01-50$ & & \\
\hline & $\mathrm{B}_{3}$ & Gradient elution $30 \mathrm{~min}$ & & & $8-10$ & $0.02-50$ & & \\
\hline & $\mathrm{B}_{5}$ & & & & 8 & $0.02-50$ & & \\
\hline & $\mathrm{B}_{6}$ & & & & $1-3$ & $0.01-50$ & & \\
\hline & $\mathrm{B}_{7}$ & & & & 3 & $0.02-50$ & & \\
\hline & $\mathrm{B}_{9}$ & & & & 9 & $0.01-50$ & & \\
\hline & $\mathrm{C}$ & & & & 12 & $0.02-50$ & & \\
\hline 5 & $\mathrm{~B}_{1}$ & A: $0.025 \%$ TFA (pH 2.6) & $\mathrm{C} 18,250 \times 4.6 \mathrm{~mm}$ & UV & - & $1.25-50$ & Infant foodstuff & Heudi et al. (2005) \\
\hline & $\mathrm{B}_{2}$ & B: $\mathrm{CH}_{3} \mathrm{CN}$ & $(5 \mu \mathrm{m})$ & & & $0.62-25$ & & \\
\hline & $\mathrm{B}_{3}$ & Gradient elution $17 \mathrm{~min}$ & & & & $1.25-50$ & & \\
\hline & $\mathrm{B}_{5}$ & & & & & $2.5-500$ & & \\
\hline & $\mathrm{B}_{6}$ & & & & & $0.125-50$ & & \\
\hline & $\mathrm{B}_{8}$ & & & & & $0.5-50$ & & \\
\hline & $\mathrm{B}_{9}$ & & & & & $0.6-25$ & & \\
\hline & $\mathrm{B}_{12}$ & & & & & $0.25-10$ & & \\
\hline & $\mathrm{C}$ & & & & & $5-200$ & & \\
\hline 6 & $\mathrm{~B}_{1}$ & $\begin{array}{l}\text { A: } 16 \mathrm{mM} \text { SDS }+0.02 \mathrm{M} \\
\text { phosphate buffer } \\
(\mathrm{pH} 3.6)+3.5 \% \mathrm{BuOH}\end{array}$ & $\begin{array}{l}\text { C18. } 150 \times 4.6 \mathrm{~mm} \\
\quad(7 \mu \mathrm{m})\end{array}$ & UV & 17,000 & $125-400$ & Vitamin preparations & Ghorbani et al. (2004) \\
\hline & $\mathrm{B}_{2}$ & $\begin{array}{l}\text { B: } 16 \mathrm{mM} \text { SDS }+0.02 \mathrm{M} \\
\text { phosphate buffer } \\
(\mathrm{pH} 3.6)+10 \% \mathrm{BuOH}\end{array}$ & $\begin{array}{l}\text { C18. } 250 \times 4.6 \mathrm{~mm} \\
\quad(10 \mu \mathrm{m})\end{array}$ & & 4,000 & $11-68$ & & \\
\hline & $\mathrm{B}_{3}$ & Gradient elution $75 \mathrm{~min}$ & & & 20,000 & $95-750$ & & \\
\hline & $\mathrm{B}_{6}$ & & & & 5,000 & $12-140$ & & \\
\hline & $\mathrm{B}_{9}$ & & & & 7,100 & $50-175$ & & \\
\hline
\end{tabular}


Table 1 (continued)

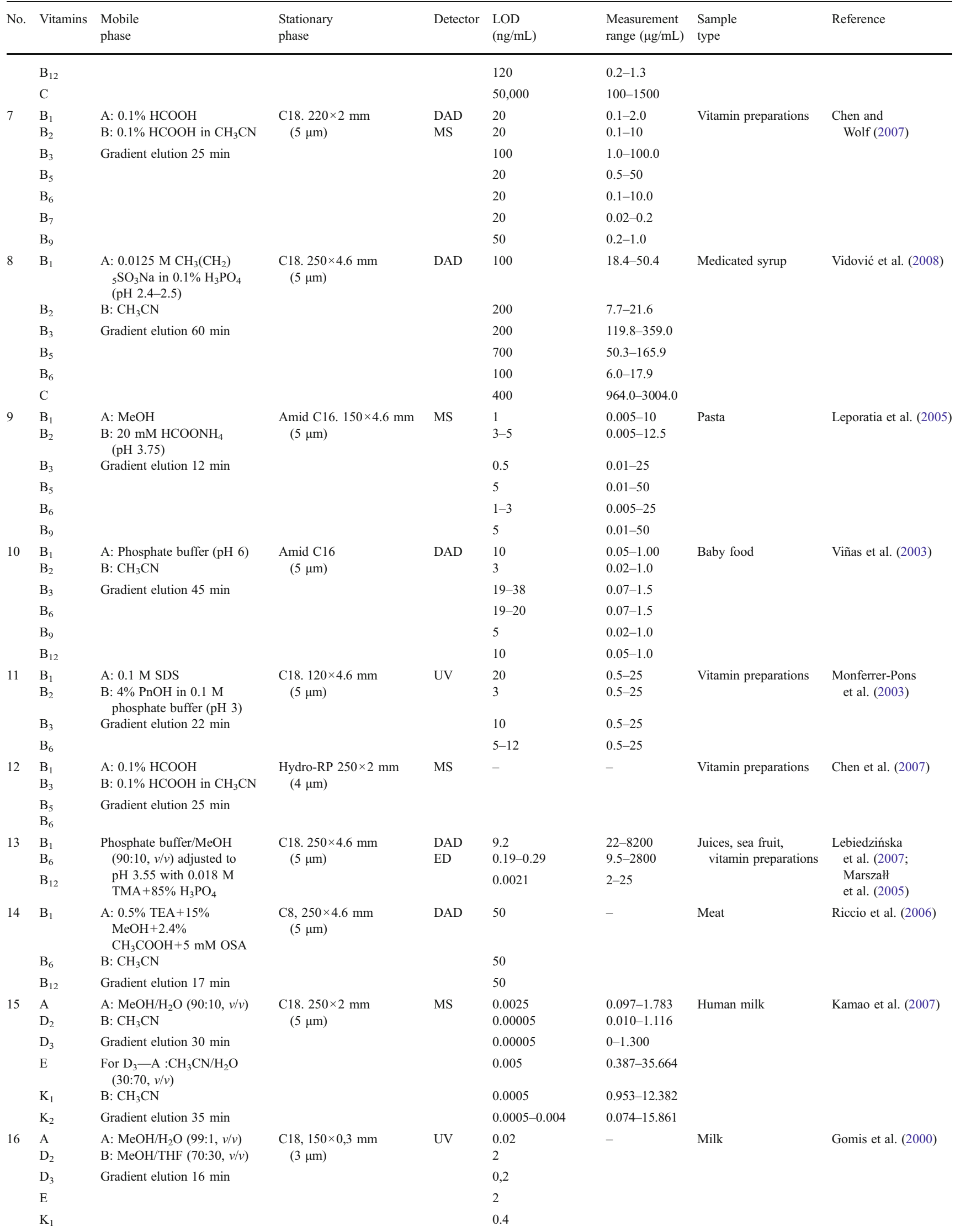


Table 1 (continued)

\begin{tabular}{|c|c|c|c|c|c|c|c|c|}
\hline No. & Vitamins & $\begin{array}{l}\text { Mobile } \\
\text { phase }\end{array}$ & $\begin{array}{l}\text { Stationary } \\
\text { phase }\end{array}$ & Detector & $\begin{array}{l}\text { LOD } \\
(\mathrm{ng} / \mathrm{mL})\end{array}$ & $\begin{array}{l}\text { Measurement } \\
\text { range }(\mu \mathrm{g} / \mathrm{mL})\end{array}$ & $\begin{array}{l}\text { Sample } \\
\text { type }\end{array}$ & Reference \\
\hline 17 & $\begin{array}{l}\mathrm{A} \\
\mathrm{D}_{3} \\
\mathrm{E} \\
\mathrm{K}_{1}\end{array}$ & $\begin{array}{l}3 \% \mathrm{SDS}+15 \% \mathrm{BuOH} \\
\text { adjusted to } \mathrm{pH} 7 \text { with } \\
0.02 \mathrm{M} \text { phosphate buffer }\end{array}$ & $\begin{array}{l}\mathrm{C} 18.250 \times 4.6 \mathrm{~mm} \\
\quad(5 \mu \mathrm{m})\end{array}$ & $\mathrm{DAD}$ & $\begin{array}{l}810 \\
910 \\
1120 \\
830\end{array}$ & $\begin{array}{l}1.0-60 \\
1.0-40 \\
1.0-60 \\
1.0-60\end{array}$ & $\begin{array}{r}\text { Food, vitamin } \\
\text { preparations }\end{array}$ & Kienen et al. (2008) \\
\hline 18 & $\begin{array}{l}\mathrm{A} \\
\mathrm{D}_{2} \\
\mathrm{D}_{3} \\
\mathrm{E}\end{array}$ & $\mathrm{MeOH} / \mathrm{H}_{2} \mathrm{O}(99: 1, v / v)$ & $\begin{array}{l}\mathrm{C} 18,120 \times 2 \mathrm{~mm} \\
\quad(5 \mu \mathrm{m})\end{array}$ & UV-Vis & - & - & Vitamin preparations & Kozlov et al. (2003) \\
\hline 19 & $\begin{array}{l}\mathrm{A} \\
\mathrm{C} \\
\mathrm{E}\end{array}$ & $\mathrm{MeOH}$ & $\begin{array}{l}\mathrm{C} 18,125 \times 4 \mathrm{~mm} \\
\quad(5 \mu \mathrm{m})\end{array}$ & UV-Vis & $\begin{array}{l}15-36 \\
65 \\
53\end{array}$ & $\begin{array}{l}1.6-7.7 \\
5.0-100.7 \\
0.04-0.19\end{array}$ & Vitamin preparations & Paulo et al. (1999) \\
\hline 20 & $\begin{array}{l}\mathrm{A} \\
\mathrm{D}_{3} \\
\mathrm{E}\end{array}$ & $\begin{array}{l}\text { Heksan }+ \text { dioksan }+\mathrm{i}-\mathrm{PrOH} \\
\quad(96.7: 3: 0.3, v / v / v)\end{array}$ & $\mathrm{C} 18250 \times 4.6 \mathrm{~mm}$ & MS & $\begin{array}{l}0.0014 \\
0.000008 \\
0.00025\end{array}$ & $\begin{array}{l}0.15-12 \\
0.005-0.4 \\
0.25-20\end{array}$ & Infant nutrition & Heudi et al. (2004) \\
\hline 21 & $\begin{array}{l}\mathrm{A} \\
\mathrm{E} \\
\mathrm{K}_{1}\end{array}$ & $\mathrm{MeOH} / \mathrm{H}_{2} \mathrm{O}(50: 50, v / v)$ & C18. Monolith $270 \times 10 \mathrm{~mm}$ & UV-Vis & $\begin{array}{l}3.9 \\
173 \\
16.4\end{array}$ & $\begin{array}{l}0.2-2 \\
1-10 \\
0.2-2\end{array}$ & Cereals & Xu and Jia (2009) \\
\hline 22 & $\begin{array}{l}\mathrm{D}_{3} \\
\mathrm{E}\end{array}$ & $\mathrm{CH}_{3} \mathrm{CN}$ with $\mathrm{Chl}+\mathrm{H}_{2} \mathrm{TPP}$ & $\begin{array}{l}\mathrm{C} 18.300 \times 3.9 \mathrm{~mm} \\
\quad(5 \mu \mathrm{m})\end{array}$ & UV & $\begin{array}{l}3.5-2300 \\
120\end{array}$ & - & Vitamin preparations & Lazareva et al. (2002) \\
\hline 23 & $\begin{array}{l}\mathrm{A} \\
\mathrm{E}\end{array}$ & $\mathrm{MeOH} / \mathrm{H}_{2} \mathrm{O}(96: 4, v / v)$ & $\begin{array}{l}\mathrm{C} 18,250 \times 4 \mathrm{~mm}(5 \mu \mathrm{m}) \\
\text { RP- } 60 \text { select B } 250 \times 4 \mathrm{~mm} \\
\quad(5 \mu \mathrm{m})\end{array}$ & $\begin{array}{l}\text { UV } \\
\text { FD }\end{array}$ & - & - & $\begin{array}{l}\text { Meat, milk, powdered } \\
\text { milk }\end{array}$ & Berg et al. (2000) \\
\hline 24 & $\begin{array}{l}\mathrm{A} \\
\mathrm{E}\end{array}$ & $\begin{array}{l}\mathrm{A}: \mathrm{CH}_{3} \mathrm{CN} / 0.04 \% \text { TFA }(1: 1, v / v) \\
\mathrm{B}: \mathrm{CH}_{3} \mathrm{CN} / \mathrm{MeOH}(3: 7, v / v) \\
\text { Gadient elution } 15 \mathrm{~min}\end{array}$ & $\begin{array}{l}\mathrm{C} 18,120 \times 2 \mathrm{~mm} \\
\quad(5 \mu \mathrm{m})\end{array}$ & UV & - & - & Vitamin preparations & Kozlov et al. (2004) \\
\hline 25 & $\begin{array}{l}\mathrm{A} \\
\mathrm{E}\end{array}$ & $\begin{array}{l}\text { A: } \mathrm{MeOH} / \mathrm{H}_{2} \mathrm{O}(96: 4, v / v) \\
\mathrm{B}: \mathrm{TBME} / \mathrm{MeOH} / \mathrm{H}_{2} \mathrm{O} \\
\quad(90: 6: 4, v / v / v) \\
\text { Gradient elution } 30 \mathrm{~min}\end{array}$ & $\begin{array}{l}\mathrm{C} 30.250 \times 4.6 \mathrm{~mm} \\
\quad(5 \mu \mathrm{m})\end{array}$ & $\begin{array}{l}\text { DAD } \\
\text { MS }\end{array}$ & $\begin{array}{l}100-1,100(\mathrm{LOQ}) \\
100-10,000(\mathrm{LOQ})\end{array}$ & $\begin{array}{l}10-400 \\
10-400\end{array}$ & Vitamin preparations & $\begin{array}{r}\text { Breithaupt and } \\
\text { Kraut (2006) }\end{array}$ \\
\hline 26 & $\begin{array}{l}\mathrm{A} \\
\mathrm{E}\end{array}$ & $\mathrm{MeOH} / \mathrm{H}_{2} \mathrm{O}(96: 4, v / v)$ & $\begin{array}{l}\mathrm{C} 18,250 \times 4 \mathrm{~mm} \\
\quad(5 \mu \mathrm{m})\end{array}$ & UV & - & - & & $\begin{array}{l}\text { Turner and } \\
\text { Mathiasson (2000) }\end{array}$ \\
\hline 27 & $\begin{array}{l}\mathrm{A} \\
\mathrm{E}\end{array}$ & $\mathrm{MeOH}$ & $\begin{array}{l}\mathrm{C} 18,250 \times 4.6 \mathrm{~mm} \\
\quad(5 \mu \mathrm{m})\end{array}$ & DAD & $\begin{array}{l}0.33 \\
3.2-32.9\end{array}$ & $\begin{array}{l}5-100 \\
5-100\end{array}$ & Milk & $\begin{array}{l}\text { Rodas Mendoza } \\
\text { et al. (2003) }\end{array}$ \\
\hline 28 & $\begin{array}{l}\mathrm{A} \\
\mathrm{E}\end{array}$ & $\begin{array}{l}0.5 \% \mathrm{CH}_{3} \mathrm{COOC}_{2} \mathrm{H}_{5} / \text { hexane } \\
\quad(1: 1, v / v)\end{array}$ & $50 \times 2.1 \mathrm{~mm}(3 \mu \mathrm{m})$ & DAD & $\begin{array}{l}0.2-0.3 \\
4.9-8.8\end{array}$ & $\begin{array}{l}0.2-5 \\
1-100\end{array}$ & Baby food & $\begin{array}{l}\text { Chávez-Servín } \\
\text { et al. (2006) }\end{array}$ \\
\hline 29 & $\begin{array}{l}\mathrm{A} \\
\mathrm{E}\end{array}$ & $\mathrm{MeOH} / \mathrm{H}_{2} \mathrm{O}(96: 4, v / v)$ & $\begin{array}{l}\mathrm{C} 18,250 \times 4.6 \mathrm{~mm} \\
\quad(5 \mu \mathrm{m})\end{array}$ & UV & $\begin{array}{l}189 / 100 \mathrm{~g} \\
8,333 / 100 \mathrm{~g}\end{array}$ & $\begin{array}{l}0.04-3.73 \\
5.5-550\end{array}$ & $\begin{array}{l}\text { Cooked meals, milk } \\
\text { and milk products }\end{array}$ & Escrivá et al. (2002) \\
\hline 30 & $\begin{array}{l}\mathrm{A} \\
\mathrm{E}\end{array}$ & $\begin{array}{l}76.9 \mathrm{mM} \text { SDS } / 1-\mathrm{BuOH} \\
(88.3: 11.7, v / v) \text { adjusted } \\
\text { to } \mathrm{pH} 6.37 \text { with } 0.02 \mathrm{M} \\
\text { phosphate buffer }\end{array}$ & $\begin{array}{l}\mathrm{C} 18,100 \times 3.9 \mathrm{~mm} \\
\quad(5 \mu \mathrm{m})\end{array}$ & UV & $\begin{array}{l}1,710 \\
4,520\end{array}$ & $\begin{array}{l}150-1,500 \\
2,000-8,000\end{array}$ & Medicated syrup & $\begin{array}{l}\text { Momenbeik } \\
\text { et al. (2005) }\end{array}$ \\
\hline 31 & $\begin{array}{l}\mathrm{A} \\
\mathrm{E}\end{array}$ & $\begin{array}{l}\text { A: } \mathrm{CH}_{3} \mathrm{CN} \\
\text { B: } \mathrm{H}_{2} \mathrm{O} \mathrm{C} / \mathrm{MeOH} \\
\text { Gradient elution } 24 \mathrm{~min}\end{array}$ & $\begin{array}{l}\mathrm{C} 8.250 \times 4.6 \mathrm{~mm} \\
\quad(5 \mu \mathrm{m})\end{array}$ & DAD & $\begin{array}{l}0.58 \\
10\end{array}$ & $\begin{array}{l}0.86-17.16 \\
0.86-32.21\end{array}$ & $\begin{array}{l}\text { Polymeric diets for } \\
\text { enteral nutrition }\end{array}$ & Kuhn et al. (2008) \\
\hline
\end{tabular}

$\mathrm{BuOH}$ butyl alcohol, $\mathrm{Chl}$ chlorophyll, EtOH ethyl alcohol, $H 2 T P P$ tetraphenylporphyrin, $H F B A$ heptafluorobutyric acid, $i-P r O H$ isopropyl alcohol, $\mathrm{MeOH}$ methyl alcohol, $\mathrm{OSA}$ 1-octanesulfonic acid, $\mathrm{PnOH}$ amyl alcohol, SDS sodium lauryl sulfate, TBME methyl tert-butyl ether, TEA 2,2',2"trihydroxytriethylamine, TFA trifluoroacetic acid, THF tetrahydrofuran, $T M A$ trimethylamine

\section{Sample Preparation}

Samples were taken from fruits (apricot, avocado), fruitvegetable juices, and multivitamin diet supplements (powders and tablets). Apricots and avocados were peeled and cut into small pieces. Two grams for each product was weighed and then intermixed with $4 \mathrm{~mL}$ of methanol. Subsequently, samples were centrifuged for $5 \mathrm{~min}$ $(2,000 \mathrm{rpm})$. The examined material was filtered using a nylon filter with a pore size of $0.45 \mu \mathrm{m}$.
One hundred milliliters of fruit-vegetable juice was taken and then $10 \mathrm{~mL}$ of Carrez I and $10 \mathrm{~mL}$ of Carrez II solutions were added for sedimentation and subsequently centrifuged for $10 \mathrm{~min}(7,000 \mathrm{rpm})$. The examined material was filtered using a nylon filter with a pore size of $0.45 \mu \mathrm{m}$, and then solid phase extraction (SPE) was performed. A RP18 Bakerbond column (500 mg, $3 \mathrm{~mL}$ ) was used. The column was conditioned with methanol $(1 \times 3 \mathrm{~mL})$ and water $(1 \times$ $3 \mathrm{~mL}$ ). Analytes were eluted with portions of the solvents: $3 \mathrm{~mL}$ of $60 \%$ methanol in water, $3 \mathrm{~mL}$ of methanol, and 
Table 2 Gradient elution parameters

\begin{tabular}{lccc}
\hline Time $(\min )$ & A (\%) & B (\%) & Flow rate $(\mathrm{mL} / \mathrm{min})$ \\
\hline 0 & 95 & 5 & 0.6 \\
4 & 95 & 5 & 0.7 \\
10 & 2 & 98 & 0.7 \\
13 & 2 & 98 & 0.7 \\
15 & 0 & 100 & 1.3 \\
25 & 0 & 100 & 1.3 \\
\hline
\end{tabular}

$\mathrm{A}-0.01 \%$ TFA in water; $\mathrm{B}-$ methanol

$3 \mathrm{~mL}$ of chloroform. The SPE procedure was based on vitamins from multivitamin preparations extraction method (Kozhanova et al. 2002).

Two grams of diet supplements (tablet or powder) were ground, diluted in methanol, and filtered using a nylon filter with a pore size of $0.2 \mu \mathrm{m}$. Volumes of methanol used for dilution were matched with declared contents of analytes in the supplement. The volume of the filtered solution for every injection was $20 \mu \mathrm{L}$. This volume was taken three consecutive times from each analyzed sample. Matrices for standards which were used for calibration curves were prepared in the same manner as the samples.

\section{Results and Discussion}

The developed chromatographic system allows the simultaneous determination of 11 vitamins (both water- and fatsoluble). Dissociation of analytes was determined by a $\mathrm{p} K_{\mathrm{a}}$ value for each compound and mobile phase $\mathrm{pH}$ value. Thus, a solution of TFA with the proper $\mathrm{pH}$ value was used. The $\mathrm{pH}$ value of the mobile phase was chosen with $\mathrm{p} K_{\mathrm{a}}$ values of particular analytes taken into consideration to allow analyte separation. The $\mathrm{pH}$ value for trifluoroacetic acid with concentration of $0.01 \%$ is equal to 3 . At the start
Table 4 Retention parameters for water- and fat-soluble vitamins

\begin{tabular}{lrrrcc}
\hline Compound & \multicolumn{1}{l}{$k^{\prime}$} & \multicolumn{1}{l}{$R$} & $\alpha$ & LOD $(\mu \mathrm{g} / \mathrm{mL})$ & LOQ $(\mu \mathrm{g} / \mathrm{mL})$ \\
\hline $\mathrm{B}_{1}$ & 3.35 & 0.93 & 1.01 & 0.96 & 2.90 \\
$\mathrm{C}$ & 4.50 & 2.05 & 1.44 & 0.90 & 2.70 \\
$\mathrm{~B}_{6}$ & 8.49 & 16.73 & 1.67 & 0.24 & 0.72 \\
$\mathrm{~B}_{3}$ & 8.98 & 4.05 & 1.36 & 0.23 & 0.70 \\
$\mathrm{~B}_{5}$ & 12.47 & 8.08 & 1.32 & 0.30 & 0.90 \\
$\mathrm{~B}_{12}$ & 13.19 & 1.91 & 1.07 & 0.41 & 1.22 \\
$\mathrm{~B}_{9}$ & 13.39 & 1.40 & 1.05 & 0.85 & 2.55 \\
$\mathrm{~B}_{2}$ & 13.99 & 3.49 & 1.03 & 0.83 & 2.51 \\
$\mathrm{~A}$ & 19.10 & 41.23 & 1.35 & 1.66 & 4.98 \\
$\mathrm{D}_{3}$ & 22.95 & 51.65 & 1.73 & 5.28 & 15.84 \\
$\mathrm{E}$ & 24.04 & 5.26 & 1.81 & 0.61 & 1.85 \\
\hline
\end{tabular}

of the mobile phase, gradient proportion of this acid to methanol is $95: 5(v / v)$.

With regard to the non-polar stationary phase, $\mathrm{B}_{1}$ and $\mathrm{C}$ vitamins eluted first from the chromatographic column. The $\mathrm{p} K_{\mathrm{a}}$ values of those compounds are 3.8 and 4.2 , respectively. Applying a $0.01 \%$ solution of TFA allowed increasing the affinity of the compounds to the stationary phase in the initial part of the analysis. It allowed one to determine and elute outside of the dwell time of vitamin $B_{1}$ and $C$. Both flow rate of the mobile phase and temperature of the stationary phase column were also subject to examinations. Optimal analytical conditions were obtained at a temperature of $30^{\circ} \mathrm{C}$. The flow rate gradient shown in Table 2 was applied. Detection was performed with the use of a diode array detector. Thanks to this, it was possible to optimize the parameters of analytical wavelengths, which allowed decreasing interferences from the matrix. Calibration curve parameters are shown in Table 3.

The retention parameters obtained for particular analytes, capacity factor $\left(k^{\prime}\right)$, selectivity $(\alpha)$, and limits of detection
Table 3 Retention times and calibration curve parameters for vitamins $(n=6)$

\begin{tabular}{lrrrrrrr}
\hline Compound & $\begin{array}{l}\text { Retention times } \\
(\mathrm{min})\end{array}$ & Slope & Intercept & Sa & Sb & $\begin{array}{l}\text { Measurement } \\
\text { range }(\mu \mathrm{g} / \mathrm{mL})\end{array}$ & $R^{2}$ \\
\hline $\mathrm{B}_{1}$ & 3.36 & 5,632 & 5,407 & 34 & 1,060 & $11-86$ & 0.997 \\
$\mathrm{C}$ & 4.51 & 2,259 & $-28,444$ & 68 & 2,119 & $80-480$ & 0.996 \\
$\mathrm{~B}_{6}$ & 8.51 & 7,729 & 235,405 & 282 & 37,424 & $34-204$ & 0.995 \\
$\mathrm{~B}_{3}$ & 8.99 & 2,293 & $-691,107$ & 89 & 66,487 & $432-936$ & 0.994 \\
$\mathrm{~B}_{5}$ & 12.48 & 1,572 & 13,685 & 37 & 1,193 & $204-408$ & 0.998 \\
$\mathrm{~B}_{12}$ & 13.20 & 20,253 & $-324,681$ & 899 & 34,693 & $27-48$ & 0.994 \\
$\mathrm{~B}_{9}$ & 13.41 & 34,595 & $-163,720$ & 1,082 & 28,866 & $17-34$ & 0.996 \\
$\mathrm{~B}_{2}$ & 14.00 & 15,516 & $-94,937$ & 636 & 34,597 & $35-70$ & 0.993 \\
$\mathrm{~A}$ & 19.12 & 261 & $-5,492$ & 3 & 306 & $27-108$ & 0.999 \\
$\mathrm{D}_{3}$ & 22.96 & 13,556 & 533,484 & 476 & 51,764 & $70-141$ & 0.995 \\
$\mathrm{E}$ & 24.05 & 1,549 & $-16,579$ & 66 & 1,072 & $105-210$ & 0.993 \\
\hline
\end{tabular}


Fig. 1 a Chromatogram of multivitamin diet supplement solution. b Chromatogram of apricot extract. c Chromatogram of fruit-vegetable juice extract a

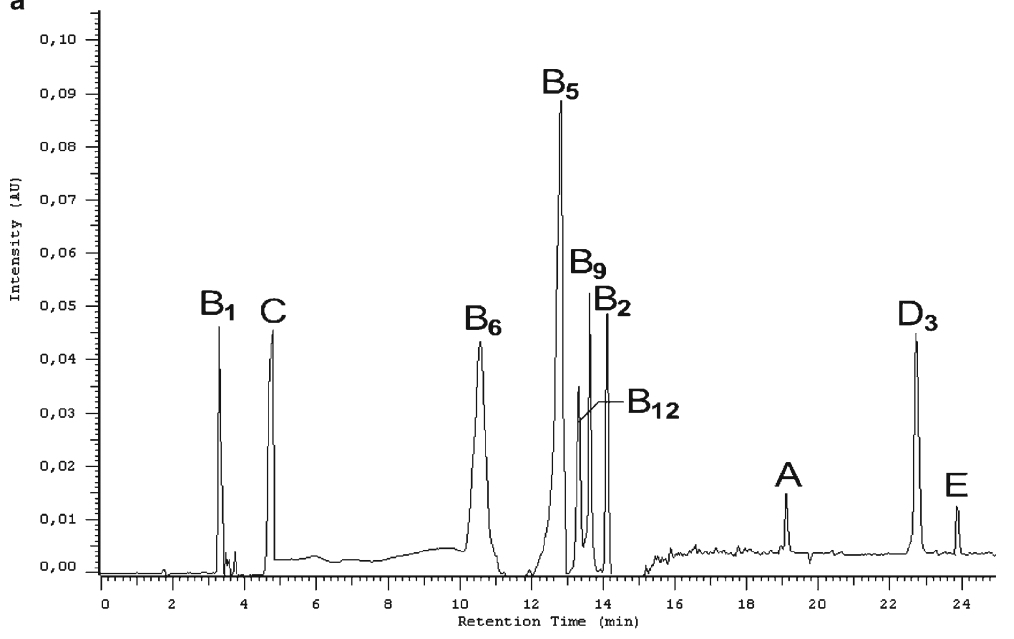

b
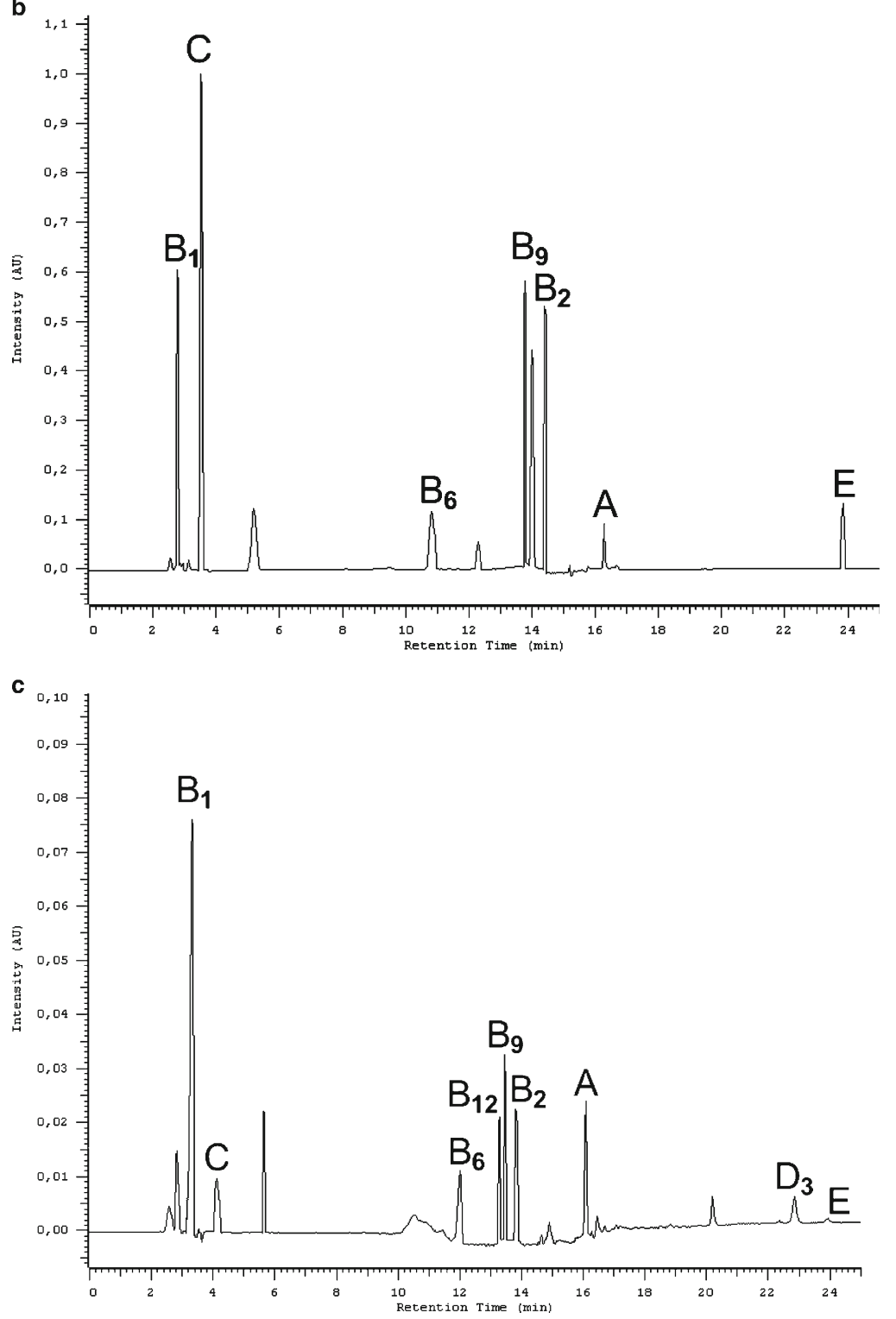
Table 5 Precision for vitamins from fruit matrix using SLE

\begin{tabular}{|c|c|c|c|c|c|c|c|c|c|}
\hline \multirow[t]{2}{*}{ Compound } & \multirow{2}{*}{$\begin{array}{l}\text { Input } \mu \mathrm{g} / \\
100 \mathrm{~g}\end{array}$} & \multicolumn{4}{|l|}{ Avocado } & \multicolumn{4}{|l|}{ Apricot } \\
\hline & & $\begin{array}{l}\text { Measured } \mu \mathrm{g} / \\
100 \mathrm{~g}\end{array}$ & $\begin{array}{l}\mathrm{SD} \mu \mathrm{g} / \\
100 \mathrm{~g}\end{array}$ & $\begin{array}{l}\text { CV } \\
(\%)\end{array}$ & $\begin{array}{l}\text { Recovery } \\
(\%)\end{array}$ & $\begin{array}{l}\text { Measured } \mu \mathrm{g} / \\
100 \mathrm{~g}\end{array}$ & $\begin{array}{l}\mathrm{SD} \mu \mathrm{g} / \\
100 \mathrm{~g}\end{array}$ & $\begin{array}{l}\mathrm{CV} \\
(\%)\end{array}$ & $\begin{array}{l}\text { Recovery } \\
(\%)\end{array}$ \\
\hline $\mathrm{B}_{1}$ & 85 & 79 & 1.44 & 2 & 93 & 68 & 1.48 & 2 & 80 \\
\hline $\mathrm{C}$ & 355 & 305 & 6.15 & 3 & 86 & 311 & 6.30 & 3 & 87 \\
\hline $\mathrm{B}_{6}$ & 61 & 51 & 2.20 & 1 & 84 & 54 & 2.05 & 1 & 88 \\
\hline $\mathrm{B}_{3}$ & 432 & 382 & 4.30 & 1 & 88 & 404 & 4.45 & 1 & 93 \\
\hline $\mathrm{B}_{12}$ & 28 & - & & & & 22 & 0.64 & 2 & 78 \\
\hline $\mathrm{B}_{9}$ & 20 & 17 & 0.86 & 2 & 83 & 18 & 0.80 & 2 & 90 \\
\hline $\mathrm{B}_{2}$ & 41 & 37 & 2.28 & 3 & 91 & 37 & 2.60 & 3 & 90 \\
\hline A & 58 & 48 & 2.70 & 3 & 83 & 50 & 2.90 & 3 & 86 \\
\hline $\mathrm{D}_{3}$ & 127 & 114 & 1.55 & 1 & 90 & 102 & 1.21 & 1 & 80 \\
\hline $\mathrm{E}$ & 110 & 91 & 3.79 & 3 & 83 & 88 & 3.81 & 3 & 80 \\
\hline
\end{tabular}

(LOD) and quantification (LOQ) values, are shown in Table 4. Limits of detection and quantification values were determined from the signal-to-noise ratio on the assumption that the signal should be three times more intensive than the noise and the LOQ is three times higher than the LOD.

Comparing the obtained LOD and LOQ values with the literature, it can be noticed that in the works which present the application of the same detector, the LOD and LOQ values are slightly lower or comparable (Ghorbani et al. 2004; Vidović et al. 2008; Leporatia et al. 2005). However, these chromatographic systems do not allow the simultaneous determination of such number of analytes. It should also be noted that the obtained LOD and LOQ values are sufficient for the determination of the examined compounds in fruits and juices as well as in diet supplements.

The obtained selectivity factor $(\alpha)$ values above 1 (Table 4 ) mean total separation of signals for particular vitamins. The obtained LOD and LOQ values were in the range from 0.7 to $2.9 \mu \mathrm{g}$ in $1 \mathrm{~mL}$ of sample for water-soluble vitamins and from
1.85 to $15.84 \mu \mathrm{g}$ in $1 \mathrm{~mL}$ of sample for fat-soluble vitamins. When comparing the obtained LOD values with those from the literature for $\mathrm{DAD}$, one can see that the developed chromatographic system has suitable sensitivity and can be used for the determination of all examined compounds in the chosen food and multivitamin diet supplement samples. Figure 1a shows a chromatogram example of methanol solution of a diet supplement.

Procedures for the simultaneous extraction of both fatsoluble and water-soluble vitamins from fruit and juice samples were developed. Fresh fruits were purchased in stores in Poland, multivitamin juice came from the domestic market, and diet supplements were purchased from pharmacies. For analytes, the extraction from solid matrices, solidliquid extraction was used. Parameters of this extraction are given above ("Sample Preparation"). Examinations included apricot and avocado because they are rich in fat-soluble and water-soluble vitamins. Avocado samples contained nine vitamins: vitamin $C$, five vitamins of group $B\left(B_{1}, B_{3}, B_{6}\right.$,
Table 6 Precision for vitamins from juice matrix using SPE

\begin{tabular}{|c|c|c|c|c|c|}
\hline \multirow[t]{2}{*}{ Compound } & \multirow[t]{2}{*}{ Input $\mu \mathrm{g} / 100 \mathrm{~g}$} & \multicolumn{4}{|l|}{ Multivitamins juice } \\
\hline & & Measured $\mu \mathrm{g} / 100 \mathrm{~g}$ & $\mathrm{SD} \mu \mathrm{g} / 100 \mathrm{~g}$ & CV (\%) & Recovery (\%) \\
\hline $\mathrm{B}_{1}$ & 80 & 75 & 1.42 & 3 & 94 \\
\hline $\mathrm{C}$ & 355 & 299 & 6.56 & 4 & 84 \\
\hline $\mathrm{B}_{6}$ & 61 & 56 & 3.20 & 4 & 92 \\
\hline $\mathrm{B}_{3}$ & 432 & 360 & 5.10 & 3 & 83 \\
\hline $\mathrm{B}_{9}$ & 18 & 17 & 0.91 & 2 & 94 \\
\hline $\mathrm{B}_{2}$ & 41 & 37 & 2.66 & 2 & 91 \\
\hline A & 58 & 50 & 2.05 & 3 & 86 \\
\hline E & 110 & 104 & 3.70 & 2 & 95 \\
\hline
\end{tabular}


$\mathrm{B}_{9}, \mathrm{~B}_{2}$ ), and $\mathrm{A}, \mathrm{D}_{3}$, and $\mathrm{E}$ vitamins. Apricot samples additionally contained vitamin $\mathrm{B}_{12}$.

Samples of fruit-vegetable juices were prepared according to the procedure specified above ("Sample Preparation"). Solid phase extraction procedure allowed extracting all eight vitamins (water-soluble $\mathrm{C}, \mathrm{B}_{1}, \mathrm{~B}_{3}, \mathrm{~B}_{6}$, $\mathrm{B}_{9}$, and $\mathrm{B}_{2}$ as well as fat-soluble $\mathrm{A}$ and $\mathrm{E}$ ), which were present in the juices. Figure 1b, c shows a chromatogram example of apricot extract and a chromatogram of juice extract, respectively. The presence of analytes, in particular with real samples, was confirmed by comparing the absorption spectra in the range of $200-600 \mathrm{~nm}$ and adding standard solutions. Signals without labels are derived from the matrix.

Method precision was examined by analysis of recoveries from apricot, avocado, and juice matrices. The procedure for determining the value of recoveries from the solid matrix consisted of the addition of all the vitamin standards to samples of fresh fruit and then extraction with methanol. For juice samples, standard solutions were added to portions of juices, thoroughly mixed, and then representative samples were collected. Afterwards, Carrez solutions were added; the procedure was the same as for real samples. Simultaneously, whole analytical procedure for "sample without added standards" was conducted. A "sample without added standards" was a real sample of the product for which no standards solutions were added as well as no purification from the analytes was made.

The applied solid-liquid extraction (SLE) procedure achieved recoveries of water-soluble vitamins from $78 \%$ to $93 \%$ for fruit matrices. The SPE procedure achieved recoveries of water-soluble vitamins from $83 \%$ to $94 \%$ for juices matrices. For fat-soluble vitamins, recoveries were from $80 \%$ to $90 \%$ for fruits (SLE) and from $86 \%$ to $95 \%$ for multivitamin juices (SPE). Recoveries are shown in Tables 5 and 6.

Analyte contents determined in real samples of fruit, juice, and multivitamin preparation are presented in Table 7. Additionally, for the multivitamin preparation, this table contains the manufacturer's declared values. The obtained results show that the developed chromatographic procedure can be used for the simultaneous separation and determination of water- and fat-soluble vitamins in fruits, juices, and diet supplements. Problems with the determination of $\mathrm{B}_{12}$ and $\mathrm{D}_{3}$ vitamins on the levels of concentration that are present in the juices and diet supplements can be eliminated by concentrating the sample before chromatographic analysis.

\section{Conclusion}

The developed chromatographic system allows the simultaneous analysis of 11 vitamins from both groups (water- and fat-soluble) in various food samples. The procedures of sample preparation are rapid and easy to perform. They allow the simultaneous extraction of all analytes and show high recovery values. Altogether, the developed chromatographic system along with the sample preparation procedures can be routinely used in food analysis. It should be emphasized that a chromatographic system together with a sample preparation procedure allows the simultaneous determination of all examined compounds in a very short time, which has never been described before.

Table 7 Contents of water- and fat-soluble vitamins in real samples of fruits, juice, and multivitamin preparation

\begin{tabular}{|c|c|c|c|c|c|c|}
\hline \multirow[t]{2}{*}{ Compound } & \multirow[t]{2}{*}{ Avocado $(\mu \mathrm{g} / 100 \mathrm{~g})$} & \multirow[t]{2}{*}{ Apricot $(\mu \mathrm{g} / 100 \mathrm{~g})$} & \multirow[t]{2}{*}{ Multivitamin juice $(\mu \mathrm{g} / 100 \mathrm{~mL})$} & \multicolumn{2}{|c|}{ Multivitamin preparation } & \multirow[t]{2}{*}{$\mathrm{SD}(\mu \mathrm{g})$} \\
\hline & & & & Measured $(\mu \mathrm{g} / \mathrm{tab})$ & Declared $(\mu \mathrm{g} / \mathrm{tab})$ & \\
\hline $\mathrm{B}_{1}$ & 82.1 & 40.5 & 206.5 & 61.9 & 60 & 2.9 \\
\hline $\mathrm{C}$ & $1,028.6$ & 439.6 & $8,460.7$ & $2,498.2$ & 2,500 & 3.1 \\
\hline $\mathrm{B}_{6}$ & 340.1 & 12.5 & 285.1 & 58.3 & 60 & 2.5 \\
\hline $\mathrm{B}_{3}$ & $1,366.7$ & 843.7 & $2,698.2$ & 699.1 & 700 & 2.7 \\
\hline $\mathrm{B}_{5}$ & $\mathrm{nf}$ & $\mathrm{nf}$ & $\mathrm{nf}$ & 149.5 & 150 & 2.8 \\
\hline $\mathrm{B}_{12}$ & $\mathrm{nf}$ & 58.5 & $<\mathrm{LOQ}$ & $<\mathrm{LOD}$ & 0.05 & 3.0 \\
\hline $\mathrm{B}_{9}$ & 71.5 & 16.6 & 28.3 & - & - & 1.8 \\
\hline $\mathrm{B}_{2}$ & 186.3 & 62.9 & 256.9 & 68.0 & 70 & 2.6 \\
\hline A & 187.4 & 227.9 & 246.1 & 31.0 & 30 & 3.1 \\
\hline $\mathrm{D}_{3}$ & 2.8 & 7.4 & $<\mathrm{LOQ}$ & $<\mathrm{LOD}$ & 0.25 & 0.1 \\
\hline $\mathrm{E}$ & 838.9 & 184.6 & $1,480.3$ & 351.0 & 350 & 2.1 \\
\hline
\end{tabular}

$n f$ not found, $<L O D / L O Q$ below $\mathrm{LOD} / \mathrm{LOQ}$ 
Open Access This article is distributed under the terms of the Creative Commons Attribution Noncommercial License which permits any noncommercial use, distribution, and reproduction in any medium, provided the original author(s) and source are credited.

\section{References}

Berg H, Turner Ch, Dahlberg L, Mathiasson L (2000) J Biochem Biophys Methods 43:391

Breithaupt DE, Kraut S (2006) Eur Food Res Technol 222:643

Chávez-Servín JL, Castellote AI, López-Sabater MC (2006) J Chromatogr A 1122:138

Chen P, Wolf WR (2007) Anal Bioanal Chem 387:2441

Chen Z, Che B, Yao S (2006) Anal Chim Acta 569:169

Chen P, Ozcan M, Wolf WR (2007) Anal Bioanal Chem 389:343

Ching LS, Mohamed S (2001) J Agric Food Chem 49:3101

Escrivá A, Esteve MJ, Farré R, Frigola A (2002) J Chromatogr A 947:313

Franke AA, Custer LJ, Arakaki C, Murphy SP (2004) J Food Compos Anal 17:1

Frenich AG, Torres MEH, Vega AB, Vidal JLM, Bolanos PP (2005) J Agric Food Chem 53:7371

Ghorbani AR, Momenbeik F, Khorasani JH, Amini MK (2004) Anal Bioanal Chem 379:439

Gil MI, Aguayo E, Kader AA (2006) J Agric Food Chem 54:4284

Gomis DB, Fernández MP, Alvarez MDG (2000) J Chromatogr A 891:109

Heudi O, Trisconi MJ, Blake ChJ (2004) J Chromatogr A 1022:115

Heudi O, Kilinç T, Fontanna P (2005) J Chromatogr A 1070:49

Hussein A, Odumeru JA, Ayanbadejo T, Faulkner H, McNab WB, Hager H, Szijarto L (2000) Food Res Int 33:131

Kamao M, Tsugawa N, Suhara Y et al (2007) J Chromatogr B 859:192

Kienen V, Costa WF, Visentainer JV, Souza NE, Oliveira CC (2008) Talanta 75:141

Klejdus B, Petrlová J, Potěšil D et al (2004) Anal Chim Acta 520:57

Kozhanova LA, Fedorova GA, Baram GI (2002) J Anal Chem 57:40

Kozlov ÉI, Solunina IA, Lyubareva ML, Nadtochii MA (2003) Pharm Chem J 37:50

Kozlov ÉI, Solunina IA, Lyubareva ML, Nadtochii MA (2004) Pharm Chem J 38:55
Kuhn M, Nakib S, De Bandt JP, Cynober L, Lod'a C (2008) J Chromatogr A $1205: 186$

Kurilich AC, Tsau GJ, Brown A et al (1999) J Agric Food Chem 47:1576

Lazareva EE, Brykina GD, Shpigun OA (2002) J Anal Chem 57:616

Lebiedzińska A, Szefer P (2006) Food Chem 95:116

Lebiedzińska A, Marszałł ML, Kuta J, Szefer P (2007) J Chromatogr A 1173:71

Leporatia A, Catellania D, Sumana M, Andreolib R, Maninib P, Niessenc WMA (2005) Anal Chim Acta 531:87

Lopez-Berenguer C, Martinez-Ballesta MC, Moreno DA, Carvajal M, Garcia-Viguera C (2009) J Agric Food Chem 57:572

Mahattanatawee K, Manthey JA, Luzio G, Talcott ST, Goodner K, Baldwin EA (2006) J Agric Food Chem 54:7355

Marszałł ML, Lebiedzińska A, Czarnowski W, Szefer P (2005) J Chromatogr A 1094:91

Momenbeik F, Momeni Z, Khorasani JH (2005) J Pharm Biomed Anal $37: 383$

Monferrer-Pons L, Capella-Peiró ME, Gil-Agusti M, Esteve-Romero J (2003) J Chromatogr A 984:223

Moreno P, Salvadó V (2000) J Chromatogr A 870:207

Munzuroglu O, Karatas F, Geckil H (2003) Food Chem 83:205

National Academy of Sciences (1989) Washington: Food and Nutrition Board

Ndaw S, Bergaentzle M, Aoude-Werner D, Hasselmann C (2000) Food Chem 71:129

Paulo MG, Cabral Marques HM, Morais JAG, Almeida AJ (1999) J Pharm Biomed Anal 21:399

Riccio F, Mennella C, Fogliano V (2006) J Pharm Biomed Anal 41:1592

Rodas Mendoza B, Morera Pons S, Castellote Bargalló AI, López-Sabater MC (2003) J Chromatogr A 1018:197

Sikorski ZE (2007) Chemia żywności. WNT, Warszawa

Singh J, Upadhyay AK, Prasad K, Bahadur A, Rai M (2007) J Food Compos Anal 20:106

Turner Ch, Mathiasson L (2000) J Chromatogr A 874:275

Vidović S, Stojanović B, Veljković J, Pražić-Arsić L, Roglić G, Manojlović D (2008) J Chromatogr A 1202:155

Viñas P, López-Erroz C, Balsalobre N, Hernández-Córdoba M (2003) J Chromatogr A 1007:77

Wall MM (2006) J Food Compos Anal 19:434

Wang H, Cao G, Prior RL (1996) J Agric Food Chem 44:701

Xu H, Jia L (2009) J Chromatogr B 877:13 